\title{
Transcriptome analysis of Listeria monocytogenes exposed to biocide stress reveals a multi-system response involving cell wall synthesis, sugar uptake, and motility
}

\section{Aidan Casey ${ }^{1}$, Edward M. Fox ${ }^{2}$, Stephan Schmitz-Esser ${ }^{3}$, Aidan Coffey ${ }^{4}$, Olivia McAuliffe ${ }^{1 *}$ and Kieran Jordan ${ }^{1}$}

${ }^{1}$ Teagasc Food Research Centre, Fermoy, Ireland

${ }^{2}$ CSIRO Animal Food and Health Sciences, Werribee, VIC, Australia

${ }^{3}$ Institute of Milk Hygiene, Department of Farm Animals and Veterinary Public Health, University of Veterinary Medicine, Vienna, Austria

${ }^{4}$ Department of Biological Sciences, Cork Institute of Technology, Cork, Ireland

Edited by:

Danilo Ercolini, Università degli Studi di Napoli Federico II, Italy

Reviewed by:

Kalliopi Rantsiou, University of Turin, Italy

Qiaobin Xiao, University of Notre Dame, USA

\section{${ }^{*}$ Correspondence:}

Olivia McAuliffe, Department of

Food Biosciences, Teagasc Food

Research Centre, Moorepark,

Fermoy, Co. Cork, Ireland

e-mail: olivia.mcauliffe@teagasc.ie
Listeria monocytogenes is a virulent food-borne pathogen most often associated with the consumption of "ready-to-eat" foods. The organism is a common contaminant of food processing plants where it may persist for extended periods of time. A commonly used approach for the control of Listeria monocytogenes in the processing environment is the application of biocides such as quaternary ammonium compounds. In this study, the transcriptomic response of a persistent strain of $L$. monocytogenes (strain 6179) on exposure to a sub-lethal concentration of the quaternary ammonium compound benzethonium chloride (BZT) was assessed. Using RNA-Seq, gene expression levels were quantified by sequencing the transcriptome of $L$. monocytogenes 6179 in the presence $(4 \mathrm{ppm})$ and absence of BZT, and mapping each data set to the sequenced genome of strain 6179. Hundreds of differentially expressed genes were identified, and subsequent analysis suggested that many biological processes such as peptidoglycan biosynthesis, bacterial chemotaxis and motility, and carbohydrate uptake, were involved in the response of $L$. monocyotogenes to the presence of BZT. The information generated in this study further contributes to our understanding of the response of bacteria to environmental stress. In addition, this study demonstrates the importance of using the bacterium's own genome as a reference when analysing RNA-Seq data.

Keywords: Listeria monocytogenes, RNA-Seq, benzethonium chloride, transcriptome, gene expression, biocide stress

\section{INTRODUCTION}

Listeria monocytogenes is a virulent food-borne pathogen that is responsible for the bacterial infection listeriosis. Listeriosis is a relatively rare disease, having an incidence of between 2-10 reported cases per million people every year in Europe (Holck and Berg, 2009), and approximately 2000 hospitalizations per annum in the United States (Guenther et al., 2009). However, it has a significantly high mortality rate of 20-30\% (Vázquez-Boland et al., 2001), making it one of the most devastating food-borne bacterial pathogens.

The main vehicle for transmission of L. monocytogenes to the human host is through the consumption of contaminated food products. L. monocytogenes is considerably more resilient than many other bacteria associated with food, being capable of multiplying at low temperatures, low $\mathrm{pH}$ and high salt concentration (Gandhi and Chikindas, 2007). These characteristics give the organism a competitive advantage in certain types of foods, particularly chilled foods that are highly processed and have a long shelf life. Due to its ubiquitous nature, L. monocytogenes is a common contaminant of food processing facilities. The organism has proven quite difficult to eradicate, and several subtypes of the bacterium are able to persistently colonize food-processing environments over extended periods of time (Fox et al., 2011a,b). This observation of persistence has very serious consequences for food safety considering that strains which can successfully persist in such environments could and often can contribute to an increased risk of cross-contamination of products. The downstream consequences of this include financial losses due to mass product recall and indeed the possibility of human infection and disease outbreak, following consumption of contaminated products (Laksanalamai et al., 2012). An in-depth study of persistent strains of $L$. monocytogenes is however quite difficult to achieve, considering that the only criterion for defining a strain as persistent is through its re-isolation from a food processing environment on numerous occasions over a prolonged period (Kastbjerg and Gram, 2009).

Control of L. monocytogenes in the food processing environment is of paramount importance to industry if the human and economic consequences of a $L$. monocytogenes outbreak are to be minimized. A common method for the control and removal of pathogenic organisms from the processing environment is through the application of quaternary ammonium compounds (QAC), which are non-corrosive, cationic agents, used frequently and in high concentrations as biocides. A study on the minimum 
inhibitory concentrations (MICs) of a QAC required to prevent growth of L. monocytogenes (Lundén et al., 2003), indicated that a QAC concentration of between 0.63 to $5.0 \mu \mathrm{g} / \mathrm{ml}$ was sufficient to prevent the bacterium from proliferating. In industry, it is commonplace to find dilutions of about $1000 \mathrm{mg} / \mathrm{L}$ being used when applying QACs to machinery for disinfection (Meyer, 2006). While, in theory, the high concentration of QAC ensures complete eradication of any pathogenic bacteria from the surface of industrial equipment, L. monocytogenes has been shown to survive and adapt when exposed to sub-lethal concentrations of these disinfectants. A recent study investigated the transcriptional response of two different strains of L. monocytogenes (namely a persister isolated from cheese production environment and a non-persister isolated from cheese) on exposure to sub-lethal concentrations of the QAC, benzethonium chloride (BZT). Using a closely related genome as a reference for the study ( $L$. monocytogenes strain F6854), Fox et al identified numerous genes which exhibited a marked increase in expression levels on BZT exposure, including those involved in the cell wall reinforcement, sugar metabolism, transcription, $\mathrm{pH}$ regulation and biosynthesis of cofactors (Fox et al., 2011a,b).

The aim of this study was to assess the global response of a persistent strain of L. monocytogenes on exposure to sub-lethal concentrations of BZT using transcriptome sequencing and subsequent RNA-Seq analysis. Gene expression levels of strain 6179 were compared in the presence or absence of BZT using the 6179 genome sequence as the reference genome.

\section{MATERIALS AND METHODS mRNA ENRICHMENT FROM Listeria monocytogenes}

A persistent $L$. monocytogenes isolate from farmhouse cheese, strain 6179 , was grown statically at $14^{\circ} \mathrm{C}$ in tryptic soy broth (TSB) to early stationary phase, under two separate experimental conditions; in the presence ( $4 \mathrm{ppm})$ and absence $(0 \mathrm{ppm})$ of BZT (Sigma Aldrich, Co. Wicklow, Ireland). BZT was prepared by dissolving in TSB, filter-sterilizing the solution through a $0.45 \mu \mathrm{m}$ filter (Sarstedt, Co. Wexford, Ireland), and adding this solution to yield a final concentration of $4 \mathrm{ppm}$ BZT in the test sample. RNA was isolated using the Qiagen RNeasy midi kit (Qiagen, Crawley, West Sussex, UK) with some modifications. Basically, to each $50 \mathrm{ml}$ sample (one test and one control), two volumes of RNAprotect (Qiagen) were added. Samples were centrifuged for $20 \mathrm{~min}$ at $3074 \times \mathrm{g}$ and the resultant pellets resuspended in $500 \mu \mathrm{l} \mathrm{TE}$ buffer containing $5 \mathrm{mg} \mathrm{ml}^{-1}$ lysozyme (Sigma Aldrich). Samples were loaded onto the Qiagen RNeasy columns and centrifuged for $5 \mathrm{~min}$ at $1902 \times \mathrm{g}$. The resultant flow-through was applied to the column a second time to maximize RNA yield. For elution, $150 \mu \mathrm{l}$ of nuclease free water (MyBio, Kilkenny, Ireland) was added to each column, which were allowed to stand for $5 \mathrm{~min}$ at room temperature, before being centrifuged at $1902 \times \mathrm{g}$ for $5 \mathrm{~min}$. The eluate was reapplied to each column, columns were allowed to stand for a further $5 \mathrm{~min}$ at room temperature, before once again being centrifuged at $1902 \times \mathrm{g}$ for $5 \mathrm{~min}$. RNA purity was assessed using the Thermo Scientific NanoDrop, while RNA integrity was checked by electrophoresis. mRNA enrichment was carried out using the Ambion MicrobExpress kit (Bio-Sciences, Co. Dublin, Ireland) with the following modifications. RNA precipitations were performed using 3 volumes of $100 \%$ ethanol and 0.1 volumes of $3 \mathrm{M}$ sodium acetate, followed by incubation at $-70^{\circ} \mathrm{C}$ for $30 \mathrm{~min}$. RNA was recovered following centrifugation at a temperature of $4^{\circ} \mathrm{C}$, and at a speed of $18,400 \times \mathrm{g}$ for $45 \mathrm{~min}$. Resuspension of RNA following precipitation was done in $15 \mu \mathrm{l}$ TE buffer. For the RNA-annealing step, $10 \mu \mathrm{g}$ of RNA was used, with oligonucleotide capture being performed at $37^{\circ} \mathrm{C}$ for $30 \mathrm{~min}$. Enriched mRNA was ultimately resuspended in $25 \mu \mathrm{l}$ of nuclease-free water.

\section{TRANSCRIPTOME SEQUENCING}

Enriched mRNA samples for each of the two experimental growth conditions were sent for commercial sequencing on the Illumina sequencing platform (Source Bioscience UK Ltd, Nottingham, UK). The raw sequence data generated, containing the sequenced mRNA reads, was filtered to discard any reads that did not meet overall quality values as determined by the Illumina chastity filter (Source Bioscience UK Ltd). Output data generated from sequencing was stored in the standard FASTQ format for use as input for subsequent analysis.

\section{RNA-Seq ANALYSIS OF SEQUENCED DATA}

RNA-Seq analysis of sequence data generated was undertaken using the Arraystar QSeq application of the DNAStar Lasergene Genomics Suite (DNASTAR, Inc., Madison, USA). Experimental inputs for the analysis included the previously described FASTQ files containing sequenced reads for each experimental condition, and two Genbank files which contained reference genome and reference plasmid features for the complete sequence of strain 6179 . Sequenced reads generated for each of the two experimental conditions were mapped against the reference genome of strain 6179 using the QSeq application in order to quantify gene expression levels of the bacterium for each experimental condition. Mapped read count normalization was applied to the data based on the number of reads per kilobase of coding sequence per million mapped reads (RPKM) (Mortazavi et al., 2008).

\section{RESULTS AND DISCUSSION}

L. monocytogenes strain 6179 is a persistent strain of the $1 / 2 \mathrm{a}$ serotype originally isolated from a cheese processing environment. It is classed as a persistent strain due to the frequency at which it has been isolated from the same environment (greater than $50 \%$ of sampling dates over a 2 -year sampling period, and on multiple occasions over a period of 12 years)(Fox et al., 2011a,b). Considering the fact that strain 6179 is of the 1/2a serotype, and is one of three serotypes of the bacteria (along with $1 / 2 \mathrm{~b}$ and $4 \mathrm{~b}$ ) found to be prevalent in human foodborne illness (Kathariou, 2002), understanding the genetic basis by which strains such as 6179 are capable of surviving detergent treatment is of high value to the ready-to-eat food processing industry, given the threat it poses for product contamination. The aim of this study was to elucidate the molecular response of a L. monocytogenes strain with increased resistance to QACs on exposure to sub-lethal BZT. This knowledge will provide insights into potential mechanisms that may be used by L. monocytogenes to achieve persistence in the food processing environment, and to ultimately contribute to how $L$. monocytogenes contamination will be addressed in the future. 
This study quantified gene expression levels in strain 6179 following growth of the bacterium in the presence or absence of sub-lethal concentrations of BZT, and subsequently compared the differences in expression levels between the two states, in order to elucidate how the bacterium responds and adapts to the stress of exposure to this particular industrial detergent. In total, the analysis identified approximately 600 genes which demonstrated a 4-fold or greater change in relative expression in the BZT-treated sample compared to the control. Differential regulation of a factor of 7.5-fold or greater was observed in the case of over 141 genes (a similar figure to the ca. 100 genes reported above this threshold in the previous study by Fox et al.). The genes which exhibited the greatest change in relative expression between experimental states function in a range of different cellular processes.

Table 1 illustrates a comparative analysis between the results reported in this particular study and those reported in a similar study by Fox et al. (2011a,b). The difference in approach between this study and that of Fox et al. (2011a,b) concerned both the reference strain against which the mRNA reads were mapped, and indeed the type of software used to carry out the RNA-Seq analysis. While the study by Fox et al. (2011a,b) used a closely related genome as a reference (L. monocytogenes strain f6854), the genome of 6179 has since been fully sequenced, and was available for use as a reference genome in this study. In using strain F6854 as a reference genome, the previous study was quite valuable in identifying some of the key responses utilized by strain 6179 in order to tolerate exposure to BZT. However, it was limited in that response mechanisms that are specific to strain 6179 (or indeed mechanisms that are only shared by strain 6179 and certain other strains) would not have been detected by the RNA-Seq analysis if the reference strain chosen did not share them. Additionally, the authors believe that a greater degree of confidence in the precision and accuracy of the resulting read counts is warranted when using 6179's own genome as a reference. Therefore, certain genetic responses which did not meet the criteria for reporting in the previous study are recognized as important and worthy of reporting in this study. In this sense, using 6179's own fully sequenced genome as a reference provided a more comprehensive picture of how the bacterium responds to BZT exposure, and this is reflected in the results obtained in this study. Both of the aforementioned reference strains are persistent isolates of the same 1/2a serotype, and have a relatively similar genome size of $2.9 \mathrm{Mb}$ and $3.0 \mathrm{Mb}$ respectively. However, strain 6179 was isolated from a cheese production facility and contains a large $60 \mathrm{~kb}$ plasmid, while strain F6854 was first isolated from a turkey frankfurter processing plant, but does not contain a plasmid (Nelson et al., 2004).

The functional groups of genes for which there was a substantial difference in expression compared to controls are indicated for each of the studies (Table 1). While a considerable up-regulation in genes involved in peptidoglycan biosynthesis, fatty acid metabolism and the phosphotransferase system were observed in both studies, the marked increase in chemotaxis, flagellar assembly and cobalamin biosynthesis genes is only reported for this study, where mRNA reads for strain 6179 were mapped back to the bacterium's own fully sequenced genome. Relative expression levels of genes on the large $60 \mathrm{~kb}$ plasmid were also assessed, with the only noteworthy changes including an
Table 1 | Functional Groups containing genes that were reported to be considerably up-regulated upon exposure of the bacterium to BZT: comparison of this study with that of Fox et al. $(2011 a, b)$.

\begin{tabular}{|c|c|c|}
\hline \multirow[t]{2}{*}{ Functional group } & \multicolumn{2}{|c|}{ Considerable up-regulation of genes in: } \\
\hline & $\begin{array}{l}\text { This study } \\
(6179 \text { v 6179) }\end{array}$ & $\begin{array}{l}\text { Fox et al., } 2011 a, b ; \\
(\mathbf{6 1 7 9} \mathbf{v} \mathbf{6 6 8 5 4 )}\end{array}$ \\
\hline $\begin{array}{l}\text { Peptidoglycan } \\
\text { biosynthesis }\end{array}$ & + & + \\
\hline Chemotaxis & + & \\
\hline Flagellar assembly & + & \\
\hline $\begin{array}{l}\text { Fatty acid } \\
\text { metabolism/biosynthesis }\end{array}$ & + & + \\
\hline $\begin{array}{l}\text { Phosphotransferase } \\
\text { system }\end{array}$ & + & + \\
\hline $\begin{array}{l}\text { Cobalamin } \\
\text { biosynthesis }\end{array}$ & + & \\
\hline
\end{tabular}

up-regulation of the repB gene by 4.4-fold, and an up-regulation of the cadmium-transporting ATPase cadA by 2.45 -fold compared to controls.

\section{CHEMOTAXIS AND FLAGELLAR ASSEMBLY}

A number of genes involved in the signaling cascade of bacterial chemotaxis were considerably up-regulated in expression when strain 6179 was grown in the presence of BZT. Among these, a gene coding for the methyl-accepting chemotaxis protein (MCP), a transmembrane protein involved in detection and transduction of extracellular sensory signals (Derr et al., 2006), showed increased expression of greater than 6-fold compared to the control. Likewise, the two-component system, cheA/cheY, which is involved in extracellular signal transduction through the bacterial cell (Dons et al., 2004), was also up-regulated, by factors of approximately 4-fold and 7-fold respectively. cheV (11.76fold change) and cheR (7.90-fold change) were also up-regulated substantially in expression when exposed to BZT.

Genes involved in flagellar biosynthesis also showed increased levels of expression compared to the controls (Figure 1). In particular, the flaA gene which codes for flagellin, the principal component of bacterial flagellum, was up-regulated by 11.84 -fold in gene expression when 6179 was grown in the presence of the detergent. In addition to this, an up-regulation in the presence of BZT was also observed for the genes motA and motB. The MotA and MotB proteins, which together form the stator component of the flagellum itself, are integral membrane proteins that interact with the fliG, fliM and fliN genes, and serve a function in transmembrane transport of protons in the aforementioned flagellar motor complex (Tang et al., 1996). This transport of protons provides the energy required for the flagella to rotate, thus enabling the bacterial cell to become motile.

The three gene components that make up the flagellar motor switch complex, namely fliG, fliM and fliN, also exhibited an up-regulation in expression of 4.98-fold, 6.50-fold and 6.03-fold respectively. This complex (induced through interaction with the cheY gene), alters the rotation of the flagella from the default 


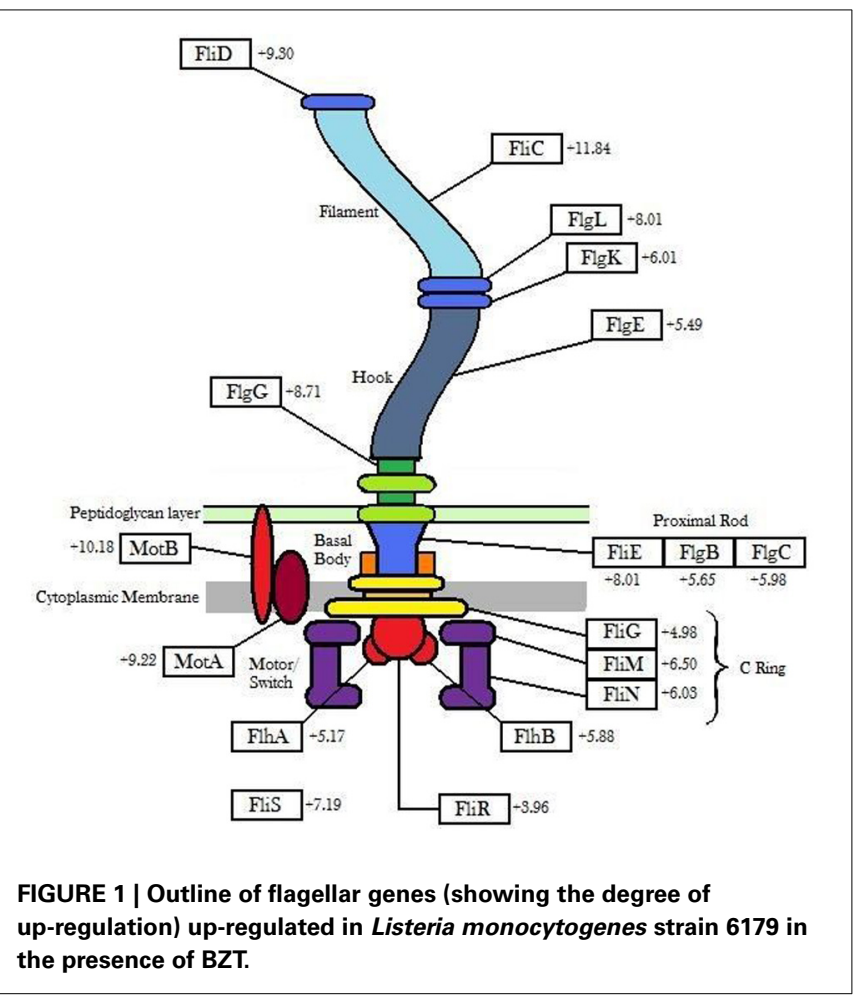

clockwise, to rotation in a counter clockwise direction. Rotation of the flagella in a clockwise direction allows the bacterial cell to experience "tumbling," whereby the cell remains in a stationary position, simply rotating in place. However, once the rotation of the flagella is altered to the counter clockwise direction, the flagella will then align at one end of the cell to form a single rotating bundle, resulting in a smooth swimming behavior, and allowing the bacteria to physically move in a desired direction (Dons et al., 2004). In this way, once the presence of detergent is detected by the organism, up-regulation of these genes will allow the bacterial cell to become motile, and move away from the unfavorable environment in which it finds itself. Other genes up-regulated in the presence of BZT (some of which, as with other subsequent tables, fall below the 4-fold change in relative expression, yet remain biologically relevant to the results) include $f l i P, f l i Q, f l i R$ and $f l i S$, as well as a number of specific flagellar structural genes such as fliD (encoding the flagellar capping protein), flgK (encoding a hookassociated protein), flgC (encoding a basal body rod protein), flgE (encoding a flagellar hook protein), flgG and flgL (Table 2).

Such a considerable up-regulation of genes associated with chemotaxis and motility had not been reported in the previous study (Fox et al., 2011a,b). This is possibly due to a combination of the reference strain used, the sensitivity of the software, and indeed the high threshold set by Fox et al. given the fact that the bacterium's own host genome was unavailable at the time. However, the response itself is somewhat logical from bacteria facing such an unfavorable environment. In a similar fashion to how bacteria will interpret and utilize extracellular signals in order to move toward a nutrient source, they will also use these signals in order to elicit a chemotactic response and allow the cell to move away from a repellent (Porter et al., 2011). The noteworthy up-regulation of the MCP gene compared to controls may suggest that the cell is in a state of constant interaction with its surroundings, reassessing the extracellular conditions as it progresses, ultimately searching for an environment where the concentration of detergent is at a more tolerable level to allow the bacterium to survive. The chemotaxis and flagellar genes work in tandem with one another in order to allow bacteria to physically respond quickly and effectively to the environmental conditions surrounding them (Hazelbauer et al., 2008).

\section{FATTY ACID BIOSYNTHESIS/METABOLISM}

This study also identified a considerable change in relative expression of a number of genes which are involved in fatty acid biosynthesis and metabolism in L. monocytogenes. In the presence of BZT, the acpP gene, which codes for the acyl carrier protein (ACP), was up-regulated by 18.25 -fold relative to expression levels observed when the bacteria were grown in the absence of the detergent. acpP belongs to a cluster of genes which are involved in the biosynthesis of fatty acids and phospholipids, a cluster which also includes the genes $p l s X, f a b D, f a b G, f a b H A$, and $f a b F$ (Martinez et al., 2010). Like $a c p P, p l s X, f a b D$, and $f a b G$ all demonstrate up-regulation in the presence of BZT (Table 3). The fabHA and $f a b F$ genes were also up-regulated; these two genes comprise the fabHAF operon, which encodes both $\mathrm{FabH}$; a protein responsible for the initiation of fatty acid elongation, and FabF, which catalyzes the remaining elongation steps in this pathway (Schujman et al., 2003).

Numerous other genes involved in fatty acid biosynthesis; fabI (4.41-fold), fabZ (3.43-fold), and fabL (3.03-fold), also exhibited an up-regulation in their expression under these conditions. In addition, genes $a c c A, a c c B, a c c C$ and $a c c D$, which together encode the four subunits of the acetyl-CoA carboxylase enzyme, were also up-regulated, by 2.37-fold, 1.79-fold, 3.52-fold, and 2.02 -fold respectively. The acetyl-CoA carboxylase enzyme is responsible for catalyzing the carboxylation of acetyl-CoA to produce malonyl-CoA, and is widely considered to be the major rate-limiting enzyme in fatty acid biosynthesis (Brownsey and Denton, 1987). The action of quaternary ammonium compounds (QAC) such as benzethonium chloride on the cell membrane of Gram-positive bacteria results in the solubilization of hydrophobic components in the membrane, such as phospholipids and lipoteichoic acids. This in turn causes a phase transition of the bacterial cell membrane from a fluid to a liquid crystalline state, and a subsequent loss of vital physiological and osmoregulatory functions of the cell itself, eventually resulting in cell death in high QAC concentrations (Gilbert and Moore, 2005). Therefore, the observation of up-regulation of genes involved in phospholipid biosynthesis when strain 6179 is exposed to BZT is somewhat expected, considering the effect that the detergent has on the fluidity of cell membrane. Previous studies (Najjar et al., 2007) have described how the membrane fluidity of L. monocytogenes can change in response to various stresses, such as that of a lowered growth temperature, and the maintenance of membrane fluidity under these stress conditions depends on the phospholipid composition within the membrane. In line with this, another gene which is indirectly involved in maintaining a fluid state of the cell 
Table 2 | Chemotaxis and flagellar assembly genes which change in regulation following exposure of strain 6179 to BZT.

\begin{tabular}{|c|c|c|c|c|}
\hline Functional category & Locus tag & Gene & Protein & Fold change \\
\hline Chemotaxis & LM6179_2457 & $M C P$ & Methyl-accepting chemotaxis protein & 6.24 \\
\hline & LM6179_1002 & cheY & Regulator of chemotaxis and motility & 6.77 \\
\hline & LM6179_1000 & cheV & Chemotaxis protein $\mathrm{CheV}$ & 11.76 \\
\hline \multirow[t]{13}{*}{ Flagellar assembly } & LM6179_1025 & fliG & Putative flagellar switch protein & 4.98 \\
\hline & LM6179_1010 & flim & Flagellar motor switch protein FliM & 6.50 \\
\hline & LM6179_1011 & fliN & Flagellar motor switch protein FliN & 6.03 \\
\hline & LM6179_1001 & $f l a A$ & Flagellin (aka FliC) & 11.84 \\
\hline & LM6179_0996 & $\operatorname{mot} A$ & Motility protein A, MotA component of the stator flagellum complex & 9.22 \\
\hline & LM6179_0997 & $\operatorname{mot} B$ & Motility protein B, MotB component of the stator flagellum complex & 10.18 \\
\hline & LM6179_1008 & $f l g E$ & Putative flagellar hook protein FlgE & 5.49 \\
\hline & LM6179_0993 & $f l g G$ & Flagellar basal body rod protein FlgG & 8.71 \\
\hline & LM6179_1017 & $f l g L$ & Flagellar hook-associated protein FlgL & 8.01 \\
\hline & LM6179_1019 & flis & Flagellar biosynthesis protein fliS & 7.19 \\
\hline & LM6179_0991 & $f \mid h A$ & Flagellar biosynthesis protein FlhA & 5.17 \\
\hline & LM6179_0990 & $f l h B$ & Flagellar biosynthesis protein FlhB & 5.88 \\
\hline & LM6179_0989 & fliR & Flagellar biosynthesis protein FliR & 3.96 \\
\hline
\end{tabular}

A gene is considered to be up-regulated if its expression level is greater when the bacterium is grown in the presence of BZT than in the absence of BZT.

Table 3 | Fatty acid biosynthesis/metabolism genes which change in regulation following exposure of strain 6179 to BZT.

\begin{tabular}{|c|c|c|c|c|}
\hline Functional category & Locus tag & Gene & Protein & Fold change \\
\hline \multirow[t]{10}{*}{ Fatty acid Biosynthesis/Metabolism } & LM6179_2576 & $a c p P$ & Acyl carrier protein & 18.25 \\
\hline & LM6179_2578 & $f a b D$ & Malonyl CoA:acyl carrier protein transacylase & 3.56 \\
\hline & LM6179_2577 & $f a b G$ & Beta-ketoacyl-acyl carrier protein reductase & 7.04 \\
\hline & LM6179_2981 & $\mathrm{fabHA}$ & Beta-ketoacyl-acyl carrier protein synthase III & 3.51 \\
\hline & LM6179_1286 & fabl & Enoyl-acyl carrier protein reductase & 4.41 \\
\hline & LM6179_1679 & $f a b Z$ & (3R)-hydroxymyristoyl-(acyl carrier protein) dehydratase & 3.43 \\
\hline & LM6179_2444 & $f a b L$ & Enoyl-acyl carrier protein reductase III & 3.03 \\
\hline & LM6179_2323 & $\operatorname{acc} A$ & Acetyl-CoA carboxylase (carboxyltransferase alpha subunit) & 2.37 \\
\hline & LM6179_2099 & $a c c B$ & Acetyl-CoA carboxylase subunit (biotin carboxyl carrier subunit) & 1.79 \\
\hline & LM6179_2100 & accC & Acetyl-CoA carboxylase subunit (biotin carboxylase subunit) & 3.52 \\
\hline
\end{tabular}

A gene is considered to be up-regulated if its expression level is greater when the bacterium is grown in the presence of BZT than in the absence of BZT.

membrane, namely fapR, was up-regulated in its expression in the presence of BZT by a factor of almost 4 -fold. This gene encodes the transcription factor FapR, which has a pivotal role in the regulation of membrane phospholipid biosynthesis by repressing the expression of a number of genes that are involved in phospholipid and fatty acid metabolism (Schujman et al., 2003).

\section{COBALAMIN BIOSYNTHESIS}

Another biosynthetic pathway that was up-regulated on exposure to BZT functions in the synthesis of cobalamin (vitamin B-12) from uroporphyrinogen III. In many Gram-positive bacteria, synthesis of cobalamin can be achieved through either an aerobic or anaerobic pathway. A different gene set is required for each 
of the pathways; the cob genes are required for the aerobic pathway, while the $c b i$ genes are required for the anaerobic pathway (Raux et al., 1998). Upon exposure of the bacteria to BZT, several of the $c b i$ genes considerably increased in their relative expression, by a range of 6 -fold to 12.5 -fold compared to controls, including the $c b i K, c b i L$, and $c b i H$ genes amongst many others (Table 4). In addition, there was also an observed increase in expression of genes up- and down-stream of the $c b i$ genes in this pathway, including $\operatorname{cobA}$ (11.09-fold change), $\operatorname{cobD}$ (9.28-fold change), and cobQ (6.25-fold change), all of which function in both the aerobic and anaerobic pathways of cobalamin biosynthesis. Although the precise reason for up-regulation of cobalamin biosynthesis is unknown, it is well documented that many bacteria, including Listeria monocytogenes (Buchrieser et al., 2003), possess genes responsible for biosynthesis of cobalamin and indeed for use of cobalamin or other closely-related intermediates of the cobalamin biosynthetic pathway as cofactors for various different biological processes, including in the degradation of ethanolamine and 1,2propanediol as a source of carbon and energy, and likewise in the synthesis of acetyl-CoA (Roth et al., 1996). In synthesizing acetylCoA, this pathway may also serve a function in feeding into the aforementioned fatty acid biosynthesis pathway, suggesting the up-regulation of these particular genes contributes to the maintenance of a functioning membrane under severe environmental stress.

\section{PEPTIDOGLYCAN BIOSYNTHESIS}

One of the most noteworthy changes observed in this study was the widespread up-regulation in the expression of genes and pathways which ultimately have a role in the biosynthesis of peptidoglycan (PG), the principal component of the Gram-positive bacterial cell wall. These include the mur family of PG biosynthesis genes (El Zoeiby et al., 2003), namely murC (1.96-fold), murD (4.44-fold), murE (4.48-fold) and murF (3.89-fold), which encode enzymes that function together with the $p d p$ (2.32-fold) and yodJ (3.16-fold) genes in the conversion
UDP-N-Acetylmuramate to peptidoglycan. Also found upregulated in the presence of BZT are genes involved in the synthesis of the UDP-N-Acetylmuramate precursor itself from fructose-6-phosphate, including gtaB (5.55-fold), glmM (4.29fold), $g \operatorname{lmS}$ (3.36-fold), and $g c a D$ (3.07-fold). The up-regulation of PG biosynthesis genes is somewhat expected considering the action of QAC's such as BZT involves disruption of the bacterial cell wall, and similar observations to these have previously been shown in the study by Fox et al. (2011a,b).

Indirectly associated with this PG biosynthesis pathway are a number of other genes which are involved in conferring structural reinforcement to the outer cell wall of L. monocytogenes. Included within this category are genes which code for proteins whose main function are in the synthesis of teichoic acids and polysaccharides. For example, the mnaA gene, which has been previously identified (Dubail et al., 2006) to play an essential role in teichoic acid biogenesis in L. monocytogenes, was up-regulated 3.64-fold in the presence of BZT. Additionally, the $\operatorname{tag} G$ and $\operatorname{tagH}$ genes, which together form the tagGH operon, were also up-regulated in the presence of the detergent, by folds of 7.11 and 10.91 respectively. This operon is homologous to that of the $\operatorname{tag} G H$ operon in Bacillus subtilis strain 168, which functions in translocation of teichoic acids through the cytoplasmic membrane (Lazarevic and Karamata, 1995). In a similar fashion, numerous other genes which also play a role in teichoic acid biosynthesis and transport are also found to increase in their relative expression under these conditions (Table 5), including ggaB (6.45-fold change), tagD (5.55-fold change), gtcA (5.42-fold change), and tagO (3.06-fold change).

Equally, a group of four genes, which together comprise the $r f b A B C D$ operon, were also up-regulated relative to controls upon exposure of strain 6179 to BZT. These genes, namely $r f b A, r f b B$, $r f b C$ and $r f b D$, exhibited an increase in relative expression by 7.25-fold, 6.46-fold, 8.85-fold, and 6.93-fold respectively, under stress. The $r f b A B C D$ operon is involved in the biosynthesis of dTDP-L-Rhamnose, which is an important component of cell

Table 4 | Cobalamin biosynthesis genes which change in regulation following exposure of strain 6179 to BZT.

\begin{tabular}{|c|c|c|c|c|}
\hline Functional category & Locus tag & Gene & Protein & Fold change \\
\hline \multicolumn{5}{|l|}{ UPREGULATED } \\
\hline \multirow[t]{14}{*}{ Cobalamin biosynthesis } & LM6179_1509 & cbik & Sirohydrochlorin cobaltochelatase & 15.24 \\
\hline & LM6179_1510 & cbil & Precorrin-2 C20-methyltransferase & 12.46 \\
\hline & LM6179_1506 & cbiH & Cobalt-precorrin-3B C(17)-methyltransferase & 8.73 \\
\hline & LM6179_1504 & cbiF & Cobalt-precorrin-4 C(11)-methyltransferase & 5.97 \\
\hline & LM6179_1505 & cbiG & CbiG protein & 7.05 \\
\hline & LM6179_1501 & cbid & Putative cobalt-precorrin-6A synthase (deacetylating) & 8.40 \\
\hline & LM6179_1507 & cbiJ/cobK & Precorrin-6X reductase & 9.35 \\
\hline & LM6179_1502 & cbiE & Putative cobalt-precorrin-6Y C(5)-methyltransferase & 10.05 \\
\hline & LM6179_1503 & cbit & Putative cobalt-precorrin-6Y C(15)-methyltransferase (decarboxylating) & 10.27 \\
\hline & LM6179_1500 & cbic & Cobalt-precorrin-8X methylmutase & 8.66 \\
\hline & LM6179_1498 & cobB/cbiA & Cobyrinic acid A,C-diamide synthase & 6.61 \\
\hline & LM6179_1508 & $\operatorname{cobA}$ & Uroporphyrin-III C-methyltransferase/uroporphyrinogen-III synthase & 11.09 \\
\hline & LM6179_1499 & $c o b D$ & Cobalamin biosynthesis protein CobD & 9.28 \\
\hline & LM6179_1515 & cobQ & Cobyric acid synthase & 6.25 \\
\hline
\end{tabular}

A gene is considered to be up-regulated if its expression level is greater when the bacterium is grown in the presence of BZT than in the absence of BZT. 
Table 5 | Peptidoglycan and teichoic acid biosynthesis genes which change in regulation following exposure of strain 6179 to BZT.

\begin{tabular}{|c|c|c|c|c|}
\hline Functional category & Locus tag & Gene & Protein & Fold change \\
\hline \multicolumn{5}{|l|}{ UPREGULATED } \\
\hline \multirow[t]{11}{*}{ Peptidoglycan biosynthesis } & LM6179_1014 & murB & UDP-N-acetylenolpyruvoylglucosamine reductase & 7.80 \\
\hline & LM6179_2355 & murC & UDP-N-acetyl muramate-alanine ligase & 1.97 \\
\hline & LM6179_2808 & murD & UDP-N-acetylmuramoylalanyl-D-glutamate ligase & 4.45 \\
\hline & LM6179_2810 & murE & $\begin{array}{l}\text { UDP-N-acetylmuramoylalanyl-D-glutamate-2, 6-diaminopimelate } \\
\text { ligase }\end{array}$ & 4.49 \\
\hline & LM6179_1168 & murF & $\begin{array}{l}\text { UDP-N-acetylmuramoylalanyl-D-glutamyl-2, } \\
\text { 6-diaminopimelate-D-alanyl-D-alanine ligase }\end{array}$ & 3.89 \\
\hline & LM6179_2811 & $p b p B$ & Penicillin-binding protein 2B & 2.33 \\
\hline & LM6179_2625 & yodJ & D-alanyl-D-alanine carboxypeptidase lipoprotein & 3.17 \\
\hline & LM6179_1398 & $g \operatorname{taB}$ & UTP-glucose-1-phosphate uridylyltransferase & 5.55 \\
\hline & LM6179_2893 & $g / m M$ & Phosphoglucosamine mutase & 4.29 \\
\hline & LM6179_1039 & glmS & $\begin{array}{l}\text { L-glutamine-D-fructose-6-phosphate amidotransferase (AKA } \\
\text { Glucosamine-fructose-6-phosphate aminotransferase) }\end{array}$ & 3.36 \\
\hline & LM6179_0488 & $g c a D$ & $\begin{array}{l}\text { Bifunctional glucosamine-1-phosphate } \\
\text { N-acetyltransferase/UDP-N-acetylglucosamine pyrophosphorylase }\end{array}$ & 3.07 \\
\hline \multirow[t]{11}{*}{ Teichoic acid biosynthesis } & LM6179_1666 & mnaA & UDP-N-acetylmannosamine 2-epimerase & 3.64 \\
\hline & LM6179_1395 & $\operatorname{tag} H$ & Teichoic acids export ATP-binding protein TagH & 10.91 \\
\hline & LM6179_1394 & $\operatorname{tag} G$ & Teichoic acid precursors permease & 7.11 \\
\hline & LM6179_1400 & $g g a B$ & Minor teichoic acids biosynthesis protein GgaB & 6.45 \\
\hline & LM6179_1409 & $\operatorname{tag} D$ & Glycerol-3-phosphate cytidylyltransferase & 5.55 \\
\hline & LM6179_1653 & gtcA & Cell wall teichoic acid glycosylation protein gtcA & 5.42 \\
\hline & LM6179_1684 & $\operatorname{tag} O$ & $\begin{array}{l}\text { UDP-N-acetylglucosamine:undecaprenyl-P N-acetylglucosaminyl-1-P } \\
\text { transferase }\end{array}$ & 3.06 \\
\hline & LM6179_1401 & rfbA & Glucose-1-phosphate thymidylyltransferase & 7.25 \\
\hline & LM6179_1403 & $r f b B$ & dTDP-glucose 4,6-dehydratase & 6.46 \\
\hline & LM6179_1402 & $r f b C$ & dTDP-4-deoxyrhamnose-3,5-epimerase & 8.85 \\
\hline & LM6179_1404 & $r f b D$ & dTDP-4-dehydrorhamnose reductase & 6.93 \\
\hline
\end{tabular}

A gene is considered to be up-regulated if its expression level is greater when the bacterium is grown in the presence of BZT than in the absence of BZT.

wall polysaccharides in many Gram-positive bacteria (Boels et al., 2004).

\section{PTS SYSTEM}

Following exposure of the bacterium to BZT, several genetic components of the phosphotransferase system (PTS) were found to be up-regulated compared to controls. The $p t s H$ and $p t s I$ genes, which together form the ptsHI operon, are up-regulated in their expression by 4.33 -fold and 4.47-fold respectively, when strain 6179 is grown in the presence of BZT. The ptsHI operon encodes two components of the phosphoenolpyruvate (PEP)-dependent PTS, namely $\operatorname{HPr}(p t s H)$ and Enzyme I ( $p t s I)$, which function in glucose uptake into the bacterial cell (Christensen et al., 1998). In addition, the $p t s G$ gene, which encodes a major glucose-specific transporter in L. monocytogenes (Stoll and Goebel, 2010) is upregulated 11.72-fold in the presence of BZT. The $b g l P H$ operon, consisting of the genes $\operatorname{bglP}$ (27.79-fold change) and $b g l H$ (11.11fold change) was also found to be up-regulated in the presence of the detergent. This operon encodes two further enzymatic components of the PEP-dependent PTS in Gram-positive bacteria (Krüger and Hecker, 1995).

Numerous other genes encoding subunits or components of the phosphotransferase system also exhibited up-regulation following exposure to BZT of a range between 20- to 70-fold in expression compared to controls (Table 6). The PTS system is responsible for the phosphorylation and translocation of sugars across the cell membrane. Upon exposure to the QAC, the bacterium responds by up-regulating a number of pathways, such as those of peptidoglycan biosynthesis, which require carbohydrates, or indeed products of carbohydrate metabolism as precursors. When strain 6179 is grown in BZT, components of the PTS-system which are specifically involved in the uptake of glucose, fructose, and mannose are up-regulated in their relative expression. Glucose and fructose are fed into the glycolysis pathway in order to be converted to fructose-6-phosphate, which ultimately serves as a specific precursor for the PG biosynthesis pathway. Consequently, an increase in expression of a number of genes involved in the glycolysis pathway is also observed upon exposure of strain 6179 to the detergent, including a 29.83 -fold up-regulation of the phosphoglyceromutase gene $g p m A$, a 5.33fold up-regulation of phosphoglucomutase gene $p g c A$, and an up-regulation of a number of genes coding for various kinases including glucose kinase $g l c K$ (4.21-fold), pyruvate kinase $p y k$ (3.31-fold), and acetate kinase ackA (2.74-fold).

\section{STRESS RESPONSE}

As expected when subjected to a harsh environment, L. monocytogenes increases expression levels of several stress response 
Table 6 | Phosphotransferase system and glycolysis genes which change in regulation following exposure of strain 6179 to BZT.

\begin{tabular}{|c|c|c|c|c|}
\hline Functional category & Locus tag & Gene & Protein & Fold change \\
\hline \multicolumn{5}{|l|}{ UPREGULATED } \\
\hline \multirow[t]{9}{*}{ Phosphotransferase system } & LM6179_1320 & ptsl & Phosphotransferase system (PTS) enzyme I & 4.47 \\
\hline & LM6179_1319 & ptsH & $\begin{array}{l}\text { Histidine-containing phosphocarrier protein of the } \\
\text { phosphotransferase system (PTS) (HPr protein) }\end{array}$ & 4.33 \\
\hline & LM6179_1334 & $p t s G$ & Putative PTS system, glucose-specific, IIA component & 11.72 \\
\hline & LM6179_0306 & $b g / P$ & PTS system beta-glucoside-specific ElIBCA component & 27.79 \\
\hline & LM6179_0618 & $b g / H$ & aryl-phospho-beta-d-glucosidase & 11.11 \\
\hline & LM6179_2766 & LM6179_2766 & PTS system protein & 69.14 \\
\hline & LM6179_2769 & LM6179_2769 & PTS system mannose/fructose/sorbose family IID subunit & 66.88 \\
\hline & LM6179_2770 & LM6179_2770 & PTS system protein & 48.23 \\
\hline & LM6179_2771 & LM6179_2771 & PTS system mannose/fructose/sorbose family IIB subunit & 21.06 \\
\hline \multirow[t]{5}{*}{ Glycolysis } & LM6179_0815 & gpmA & Phosphoglycerate mutase family protein & 29.83 \\
\hline & LM6179_2641 & $\operatorname{pgc} A$ & Phosphoglucomutase/phosphomannomutase & 5.33 \\
\hline & LM6179_2081 & glcK & Glucose kinase & 4.21 \\
\hline & LM6179_2321 & pyk & Pyruvate kinase & 3.31 \\
\hline & LM6179_2332 & $\operatorname{ack} A$ & Acetate kinase & 2.74 \\
\hline
\end{tabular}

A gene is considered to be up-regulated if its expression level is greater when the bacterium is grown in the presence of BZT than in the absence of BZT.

genes. Included in these are $\operatorname{csp} C$ (9.25-fold) and $\operatorname{csp} D$ (4.84fold) genes, both encoding cold shock proteins. Similarly, there is a notable increase in expression of a number of heat shock genes, including $r s b R A$ (4.22-fold), ftsH (3.70-fold), hslR (3.53fold), and $h s l O$ (3.40-fold), among other general stress genes such as yloU (4.76-fold) which encodes an alkaline shock protein, and liaR (3.68-fold), which encodes a regulatory protein that responds to cell stress. While some of the observations made in terms of stress response may be attributed to cross-protection, the increased expression of a number of these genes is indicative of the bacteria's attempt to adapt to the harsh new environment in which it finds itself.

\section{MULTI-DRUG RESISTANCE TRANSPORTERS}

A considerable increase in expression of numerous genes encoding multi-drug resistance transporters is also noted when this strain of L. monocytogenes is exposed to BZT. Inclusive in these is a marked 4-fold increase in expression of the quaternary ammonium compound resistance protein, $q a c H$, which has previously been identified (Muller et al., 2013) to have a pivotal role in increased resistance of $L$. monocytogenes to QAC exposure. In addition, there was a 3 -fold increase in expression of two genes, $y k k C$ and $\operatorname{lm} r B$, which encode efflux transporters, as well as an increase in expression of several other genes which are also involved in multi-drug resistance (Table 7).

\section{PHAGE/PROPHAGE}

In contrast to other genes, those encoding phage proteins were found to be considerably down-regulated when strain 6179 was exposed to the QAC. In total, over 75 genes which fall into this category were found to decrease in expression by at least 3 -fold compared to the levels seen in the control experiment. These genes include uncharacterized phage proteins, as well as structural phage proteins, such as the major tail shift protein (11.35-fold change), major head protein (7.45-fold change), tail tape measure protein (6.60-fold change) and the major capsid protein (4.98-fold change). In addition to these, functional phage proteins such as the phage holin protein (14.49-fold change), phage portal protein (5.66-fold change) and a phage-related transcriptional activator protein (3.74-fold change) were also found to be down-regulated in the presence of BZT. Although the observed down-regulation in phage gene expression is quite substantial when strain 6179 is exposed to the detergent, the reason as to why such a change occurs is still as yet relatively unknown, and further experimentation will be required in order to fully understand the underlying mechanisms at work. A study on the response of Staphylococcus aureus to a mild acid (Weinrick et al., 2004) revealed a similar effect on prophage gene expression to that seen here, suggesting that the drop in $\mathrm{pH}$ from 7.5 to 5.5 resulted in a less frequent spontaneous induction of prophage. Considering that the $\mathrm{pH}$ of a $1 \%$ aqueous solution of BZT is somewhere in the range of 4.8-5.5, the observed down-regulation of prophage genes in the test sample could potentially be attributed to the mildly acidic environment compared to the control.

\section{CONCLUDING REMARKS}

The results obtained from the transcriptome sequence analysis suggest that the response of $L$. monocytogenes to the presence of BZT is essentially two-fold; an up-regulation in genes and gene pathways which are directly or indirectly involved in cell wall biosynthesis, and equally an up-regulation in genes that code for proteins and other components which comprise the organism's chemotaxis and motility cascade. In addition to these two main responses, the bacterium also up-regulates many genes involved in maintenance of cell membrane fluidity, as well as those involved in uptake of carbohydrates, in order to adapt to the presence of BZT. Expression of genes encoding virulence factors, such as listeriolysin $\mathrm{O}$ and the internalins, remained relatively unchanged following exposure of the bacteria to BZT (data not shown). In the study by Fox et al. (2011a,b), sequenced mRNA 
Table 7 | Stress response, multi-drug resistance transporter, and phage/prophage genes which change in regulation following exposure of strain 6179 to BZT.

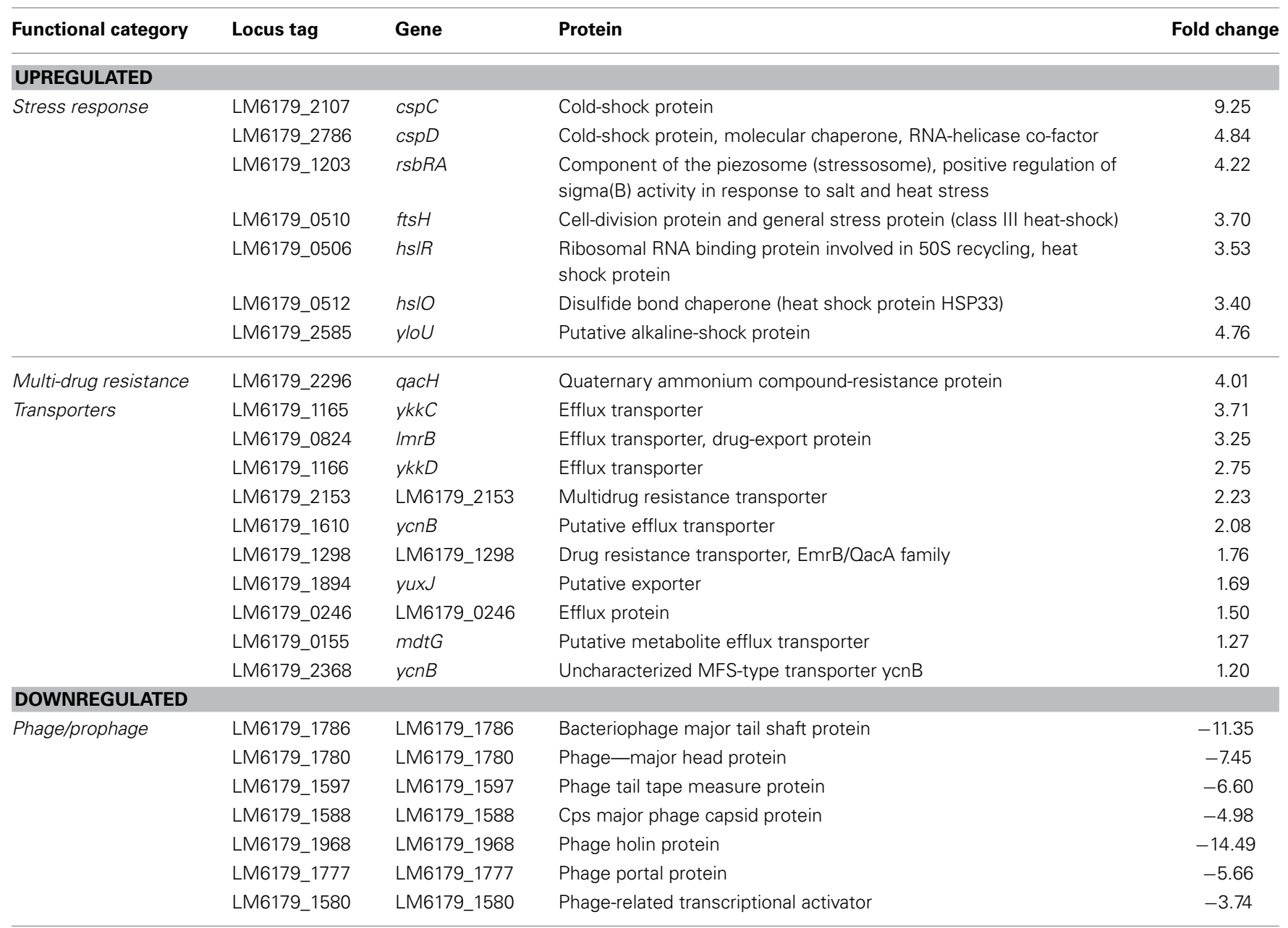

A gene is considered to be up-regulated if its expression level is greater when the bacterium is grown in the presence of BZT than in the absence of BZT.

reads from this particular strain of the bacteria were mapped and quantified against a similar reference genome, namely L. monocytogenes strain f6854. While the results of that study made some similar observations to those discussed here where peptidoglycan biosynthesis and the phosphotransferase system are concerned, the considerable up-regulation in chemotaxis and motility genes is an observation only reported in this particular study. As previously mentioned, the primary difference between the two studies concerned the reference strain used in read mapping, in that the host's own fully sequenced genome was available for this research. While using a highly similar genome as a reference for this type of work does give us some insight into the response of L. monocytogenes to such an environmental stress as exposure to BZT, it is clear from the findings that in order to fully ascertain the bacterial response, it is more favorable to use the bacterium's own genome where possible.

From a persistence perspective, it is now generally thought that a bacterium's ability to persist in a given environment can be attributed to a combination of factors, rather than a single individual trait. This study investigated the response of a persistent strain of L. monocytogenes when exposed to a sub-lethal concentration of an industrial detergent, and, while providing an insight on a genetic level as to how this strain is able to persist and adapt to the introduction of such a threat to its survival, the results are not a complete representation of the phenomenon of persistence itself. Exposure of the bacterium to such a low level of detergent in the food processing environment would only occur as a result of improper cleaning practices, considering the extremely high concentrations at which they are generally applied to equipment and surfaces. The ability of the bacterium to form a biofilm, as well as its colonization of niche areas, such as corners of machinery or other hard to reach places, can result in the bacterium being exposed to these substantially reduced detergent concentrations, and these factors may also contribute in some part to the ability of the bacterium to achieve persistence.

\section{ACKNOWLEDGMENTS}

This work was supported by the E.U. 7th framework projects PROMISE (project number 265877) and FOODSEG (project number 266061). Aidan Casey was supported by the Teagasc Walsh Fellowship programme. 


\section{REFERENCES}

Boels, I. C., Beerthuyzen, M. M., Kosters, M. H. W., Van Kaauwen, M. P. W., Kleerebezem, M., and de Vos, W. M. (2004). Identification and functional characterization of the Lactococcus lactis $\mathrm{rfb}$ operon, required for dTDP-rhamnose biosynthesis. J. Bacteriol. 186, 1239-1248. doi: 10.1128/JB.186.5.1239-1248.2004

Brownsey, R. W., and Denton, R. (1987). Acetyl-coenzyme A carboxylase. Enzymes 18, 123-146. doi: 10.1016/S1874-6047(08)60256-5

Buchrieser, C., Rusniok, C., Kunst, F., Cossart, P., and Glaser, P. (2003). Comparison of the genome sequences of Listeria monocytogenes and Listeria innocua: clues for evolution and pathogenicity. FEMS Immunol. Med. Microbiol. 35, 207-213. doi: 10.1016/S0928-8244(02)00448-0

Christensen, D. P., Benson, A. K., and Hutkins, R. W. (1998). Cloning and expression of the Listeria monocytogenes scott A ptsH and ptsI genes, coding for HPr and enzyme I, respectively, of the phosphotransferase system. Appl. Environ. Microbiol. 64, 3147-3152.

Derr, P., Boder, E., and Goulian, M. (2006). Changing the specificity of a bacterial chemoreceptor. J. Mol. Biol. 355, 923-932. doi: 10.1016/j.jmb.2005.11.025

Dons, L., Eriksson, E., Jin, Y., Rottenberg, M. E., Kristensson, K., Larsen, C. N., et al. (2004). Role of flagellin and the two-component CheA/CheY system of listeria monocytogenes in host cell invasion and virulence. Infect. Immun. 72, 3237-3244. doi: 10.1128/IAI.72.6.3237-3244.2004

Dubail, I., Bigot, A., Lazarevic, V., Soldo, B., Euphrasie, D., Dupuis, M., et al. (2006). Identification of an essential gene of listeria monocytogenes involved in teichoic acid biogenesis. J. Bacteriol. 188, 6580-6591. doi: 10.1128/JB.00771-06

El Zoeiby, A., Sanschagrin, F., and Levesque, R. C. (2003). Structure and function of the Mur enzymes: development of novel inhibitors. Mol. Microbiol. 47, 1-12. doi: 10.1046/j.1365-2958.2003.03289.x

Fox, E., Hunt, K., O’Brien, M., and Jordan, K. (2011a). Listeria monocytogenes in Irish Farmhouse cheese processing environments. Int. J. Food Microbiol. 145(Suppl. 1), S39-S45. doi: 10.1016/j.ijfoodmicro.2010.10.012

Fox, E. M., Leonard, N., and Jordan, K. (2011b). Physiological and transcriptional characterization of persistent and nonpersistent listeria monocytogenes isolates. Appl. Environ. Microbiol. 77, 6559-6569. doi: 10.1128/AEM.05529-11

Gandhi, M., and Chikindas, M. L. (2007). Listeria: a foodborne pathogen that knows how to survive. Int. J. Food Microbiol. 113, 1-15. doi: 10.1016/j.ijfoodmicro.2006.07.008

Gilbert, P., and Moore, L. E. (2005). Cationic antiseptics: diversity of action under a common epithet. J. Appl. Microbiol. 99, 703-715. doi: 10.1111/j.13652672.2005.02664.x

Guenther, S., Huwyler, D., Richard, S., and Loessner, M. J. (2009). Virulent bacteriophage for efficient biocontrol of listeria monocytogenes in ready-to-eat foods. Appl. Environ. Microbiol. 75, 93-100. doi: 10.1128/AEM.01711-08

Hazelbauer, G. L., Falke, J. J., and Parkinson, J. S. (2008). Bacterial chemoreceptors: high-performance signaling in networked arrays. Trends Biochem. Sci. 33, 9-19. doi: 10.1016/j.tibs.2007.09.014

Holck, A., and Berg, J. (2009). Inhibition of Listeria monocytogenes in cooked ham by virulent bacteriophages and protective cultures. Appl. Environ. Microbiol. 75, 6944-6946. doi: 10.1128/AEM.00926-09

Kastbjerg, V. G., and Gram, L. (2009). Model systems allowing quantification of sensitivity to disinfectants and comparison of disinfectant susceptibility of persistent and presumed nonpersistent Listeria monocytogenes. J. Appl. Microbiol. 106, 1667-1681. doi: 10.1111/j.1365-2672.2008.04134.x

Kathariou, S. (2002). Listeria monocytogenes virulence and pathogenicity, a food safety perspective. J. Food Prot. 65, 1811-1829.

Krüger, S., and Hecker, M. (1995). Regulation of the putative bglPH operon for aryl-beta-glucoside utilization in Bacillus subtilis. J. Bacteriol. 177, 5590-5597.

Laksanalamai, P., Joseph, L. A., Silk, B. J., Burall, L. S., Tarr, C. L., Gerner-Smidt, P., et al. (2012). Genomic characterization of Listeria monocytogenes strains involved in a multistate listeriosis outbreak associated with cantaloupe in US. PLoS ONE 7:e42448. doi: 10.1371/journal.pone.0042448

Lazarevic, V., and Karamata, D. (1995). The tagGH operon of Bacillus subtilis 168 encodes a two-component $\mathrm{ABC}$ transporter involved in the metabolism of two wall teichoic acids. Mol. Microbiol. 16, 345-355. doi: 10.1111/j.13652958.1995.tb02306.x

Lundén, J., Autio, T., Markkula, A., Hellström, S., and Korkeala, H. (2003). Adaptive and cross-adaptive responses of persistent and non-persistent Listeria monocytogenes strains to disinfectants. Int. J. Food Microbiol. 82, 265-272. doi: 10.1016/S0168-1605(02)00312-4

Martinez, M. A., de Mendoza, D., and Schujman, G. E. (2010). Transcriptional and functional characterization of the gene encoding acyl carrier protein in Bacillus subtilis. Microbiology 156(Pt 2), 484-495. doi: 10.1099/mic.0.033316-0

Meyer, B. (2006). Does microbial resistance to biocides create a hazard to food hygiene? Int. J. Food Microbiol. 112, 275-279. doi: 10.1016/j.ijfoodmicro.2006.04.012

Mortazavi, A., Williams, B. A., McCue, K., Schaeffer, L., and Wold, B. (2008). Mapping and quantifying mammalian transcriptomes by RNA-Seq. Nat. Methods 5, 621-628. doi: 10.1038/nmeth.1226

Muller, A., Rychli, K., Muhterem-Uyar, M., Zaiser, A., Stessl, B., Guinane, C. M., et al. (2013). Tn6188 - a novel transposon in Listeria monocytogenes responsible for tolerance to benzalkonium chloride. PLoS ONE 8:e76835. doi: 10.1371/journal.pone.0076835

Najjar, M. B., Chikindas, M., and Montville, T. J. (2007). Changes in Listeria monocytogenes membrane fluidity in response to temperature stress. Appl. Environ. Microbiol. 73, 6429-6435. doi: 10.1128/AEM.00980-07

Nelson, K. E., Fouts, D. E., Mongodin, E. F., Ravel, J., DeBoy, R. T., Kolonay, J. F., et al. (2004). Whole genome comparisons of serotype $4 \mathrm{~b}$ and $1 / 2 \mathrm{a}$ strains of the food-borne pathogen Listeria monocytogenes reveal new insights into the core genome components of this species. Nucleic Acids Res. 32, 2386-2395. doi: 10.1093/nar/gkh562

Porter, S. L., Wadhams, G. H., and Armitage, J. P. (2011). Signal processing in complex chemotaxis pathways. Nat. Rev. Microbiol. 9, 153-165. doi: 10.1038/nrmicro2505

Raux, E., Lanois, A., Warren, M. J., Rambach, A., and Thermes, C. (1998). Cobalamin (vitamin B12) biosynthesis: identification and characterization of a Bacillus megaterium cobI operon. Biochem. J. 335, 159-166.

Roth, J., Lawrence, J., and Bobik, T. (1996). Cobalamin (coenzyme B12): synthesis and biological significance. Annu. Rev. Microbiol. 50, 137-181. doi: 10.1146/annurev.micro.50.1.137

Schujman, G. E., Paoletti, L., Grossman, A. D., and de Mendoza, D. (2003). FapR, a bacterial transcription factor involved in global regulation of membrane lipid biosynthesis. Dev. Cell 4, 663-672. doi: 10.1016/S1534-5807(03)00123-0

Stoll, R., and Goebel, W. (2010). The major PEP-phosphotransferase systems (PTSs) for glucose, mannose and cellobiose of Listeria monocytogenes, and their significance for extra- and intracellular growth. Microbiology 156, 1069-1083. doi: 10.1099/mic.0.034934-0

Tang, H., Braun, T. F., and Blair, D. F. (1996). Motility protein complexes in the bacterial flagellar motor. J. Mol. Biol. 261, 209-221. doi: 10.1006/jmbi.1996.0453 Vázquez-Boland, J. A., Kuhn, M., Berche, P., Chakraborty, T., Domínguez-Bernal, G., Goebel, W., et al. (2001). Listeria pathogenesis and molecular virulence determinants. Clin. Microbiol. Rev. 14, 584-640. doi: 10.1128/CMR.14.3.584640.2001

Weinrick, B., Dunman, P. M., McAleese, F., Murphy, E., Projan, S. J., Fang, Y., et al. (2004). Effect of mild acid on gene expression in Staphylococcus aureus. J. Bacteriol. 186, 8407-8423. doi: 10.1128/JB.186.24.8407-8423.2004

Conflict of Interest Statement: The authors declare that the research was conducted in the absence of any commercial or financial relationships that could be construed as a potential conflict of interest.

Received: 18 December 2013; accepted: 07 February 2014; published online: 28 February 2014.

Citation: Casey A, Fox EM, Schmitz-Esser S, Coffey A, McAuliffe O and Jordan K (2014) Transcriptome analysis of Listeria monocytogenes exposed to biocide stress reveals a multi-system response involving cell wall synthesis, sugar uptake, and motility. Front. Microbiol. 5:68. doi: 10.3389/fmicb.2014.00068

This article was submitted to Food Microbiology, a section of the journal Frontiers in Microbiology.

Copyright (C) 2014 Casey, Fox, Schmitz-Esser, Coffey, McAuliffe and Jordan. This is an open-access article distributed under the terms of the Creative Commons Attribution License (CC BY). The use, distribution or reproduction in other forums is permitted, provided the original author(s) or licensor are credited and that the original publication in this journal is cited, in accordance with accepted academic practice. No use, distribution or reproduction is permitted which does not comply with these terms. 